\title{
A Multiaxial Variable Amplitude Fatigue Life Prediction Method Based on a Plane Per Plane Damage Assessment
}

\author{
Bianzeube Tikri ${ }^{1,}$, , Fabienne Fennec ${ }^{2}$, Bastien Weber ${ }^{3}$, Jean-Louis Robert ${ }^{2}$ \\ ${ }^{1}$ Department of Mechanical Engineering, Polytechnic University of Mongo, Mongo, Chad \\ ${ }^{2}$ Department of Mechanical Engineering, Clermont Auvergne University, Clermont-Ferrand, France \\ ${ }^{3}$ Department of Metallurgical and Materials Engineering, ARCELORMITTAL Maizieres Research, Maizieres-Les-Metz, France
}

\section{Email address:}

bitikri@gmail.com (B. Tikri)

${ }^{*}$ Corresponding author

\section{To cite this article:}

Bianzeube Tikri, Fabienne Fennec, Bastien Weber, Jean-Louis Robert. A Multiaxial Variable Amplitude Fatigue Life Prediction Method Based on a Plane Per Plane Damage Assessment. American Journal of Mechanical and Industrial Engineering.

Vol. 3, No. 4, 2018, pp. 47-54. doi: 10.11648/j.ajmie.20180304.12

Received: June 23, 2018; Accepted: July 19, 2018; Published: August 17, 2018

\begin{abstract}
A multiaxial variable amplitude fatigue life prediction method is proposed in this paper. Three main steps are distinguished. The first one concerns the counting of multiaxial cycles and uses the normal stress to a physical plane as the counting parameter. Then a multiaxial finite fatigue life criterion allows one to assess the material life corresponding to each cycle on any physical plane. A damage law and its cumulation rule describe the damage induced by each cycle plane per plane. By this way the critical plane for a given multiaxial stress history is found out. It is assumed to be the fracture plane and the fatigue life of the material is traduced as the number of repetitions of the sequence up to crack initiation. At this stage, material fatigue criteria and linear and nonlinear damage laws assume that the material is damaged. One distinguishes among these criteria critical plan type whose formalism can identify the crack initiation plan. An application is given for each load. In the context of multiaxial solicitations of variable amplitude, a validation of the estimation of the orientations of the priming planes is carried out based on experimental results on cruciform test pieces; the estimated orientations are close to those observed experimentally.
\end{abstract}

Keywords: Multiaxial Fatigue, Variable Amplitude, Fatigue Life, Damage Law, Cycle Counting

\section{Introduction}

Most mechanical structures or machine elements are nowadays fatigue-prone components. As both economical and environmental constraints lead nowadays to a decrease of material weight for some given work conditions, the materials are thus often submitted to high stress levels. In the case of variable amplitude loading, stress amplitudes are consequently larger and induce fatigue damage of materials. the durability assessment has become by this way a check point for lots of mechanical components that engineers have to design.

An accurate assessment of the level of safety of a structure requires a thorough examination of the whole components to identify and then verify their critical areas. despite the fact that for uniaxial variable amplitude or multiaxial constant amplitude loadings, some efficient tools are developed concerning cycles counting and multiaxial fatigue criteria, few adequate methods are proposed for the most general case of solicitations, i.e. the case of multiaxial variable amplitude stress states histories.

The purpose of this paper is to describe a stress based approach that allows one to assess the fatigue life of materials submitted to such a kind of loading. This work has been developed within the framework of a collaboration between SOLLAC (steel manufacturer), the research department of EDF (electric power generation) and the laboratory of Solid Mechanics of INSA-Lyon. 


\section{Principle of the Multiaxial Variable Amplitude Fatigue Life Prediction Method}

Designers had for a long time to assess the reliability of mechanical structures which are submitted to variable amplitude stress states. In the case of uniaxial variable amplitude stress histories, usual fatigue life prediction methods use cycles counting, as level cross counting or more often Rain flow counting, and damage and cumulation rules that are devoted to traduce the fatigue damage phenomenon.

For multiaxial variable amplitude stress states, few methods however are proposed today even if a more and more accurate assessment of fatigue prone components is required. Carpinteri [1] and Wang [2] proposed a method derived from low cycle fatigue that assumes the material to be more sensitive to the shear stress effect or to the normal stress effect. Zheng-Yong [3] has built a continuum damage model that defines a damaging process independently from any cycle counting. Nicholas [4] recently proposed a fatigue life assessment based on statistical parameters of the stress history. The presented method is derived from classical uniaxial stress states fatigue life assessment methods. It is based both upon the extension of a multiaxial fatigue life criterion to finite lives and the definition of a counting variable in order to identify cycles through multiaxial variable amplitude sequences.

The two main points of the method are detailed in the following sections. The counting variable that is used has to be good representative of the all components of the stress tensor and also of their real evolution versus time. The fatigue life is assessed at the point of the mechanical component where the stress states history is known by considering all the possible material planes through that point As the crack initiation is developing on one particular plane of the material, all the possible orientations of the considered plane are examined and the damage is assessed through the stresses that the loading induces on that plane. This concept is the origin of the plane per plane damage and fatigue life valuation. Two linear and non linear damage law and cumulation rules have been adapted to multiaxial stress states to allow fatigue lives assessments. The method is general, i.e. suitable for any kind of loading history. The different steps of the procedure are described on the following flow chart (figure 1).

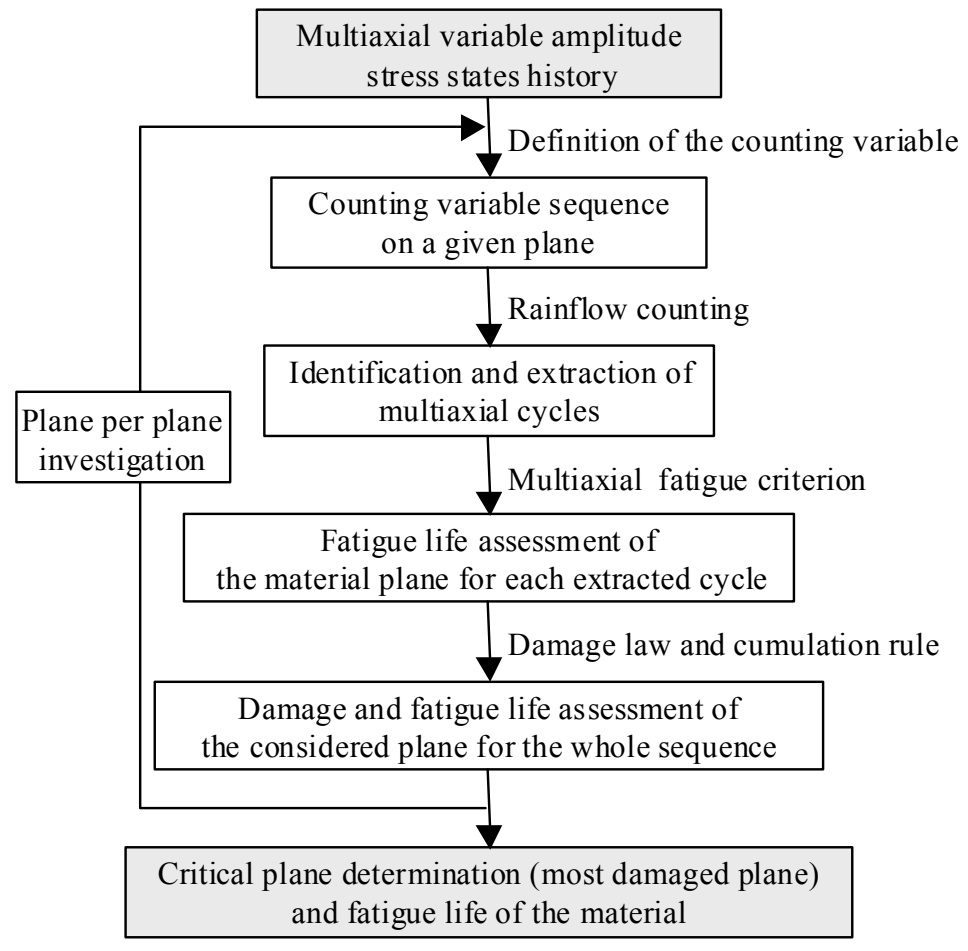

Figure 1. Flow chart of the plane per plane fatigue life assessment method.

The assumption is made that the critical plane of the material, i.e. the plane where the damage reaches the highest value, is the fracture plane of the material and consequently implies its fatigue life. The major points of the proposed method are first the counting of multiaxial cycles from a six time series history (i.e. the six components of the symmetrical stress tensor) and also the use of a multiaxial critical plane fatigue criterion to assess the damage of any multiaxial cycle. The following sections give details of these steps.

\section{Multiaxial Cycle Counting}

The Rain flow counting procedure is the emergent technique that is used today to identify stress cycles. It is based upon the appearance of a closed loop in the stressstrain material response [5]. The present purpose is not to develop the usual Rain flow technique even if the practical application may vary rather widely, despite the fact that the 
basic principles are well known. This point has led to an industrial french consensus about a standard procedure. Actually the Rain flow counting can be applied only to a uniaxial sequence. In the case of multiaxial constant amplitude stress states, the cycles counting can be correctly obtained when considering any non constant component of the stress tensor because all the components have the same frequency and as a consequence the same time period (figure 2 ). When a cycle occurs on one channel, all the other channels experience also a cycle.

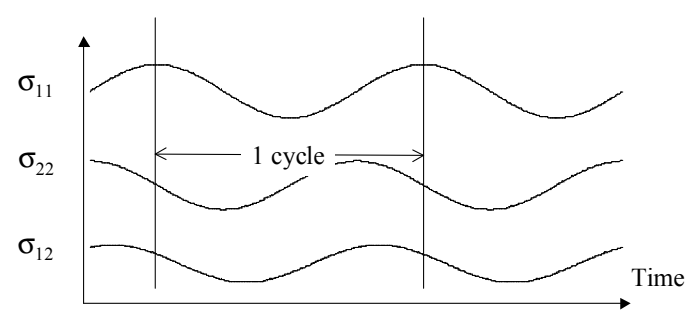

Figure 2. Identification of a cycle for a multiaxial constant amplitude stress states history.

Cycles counting are really more complex for histories of multiaxial random stress states. As a matter of fact, when all the components of the stress tensor are independent one from

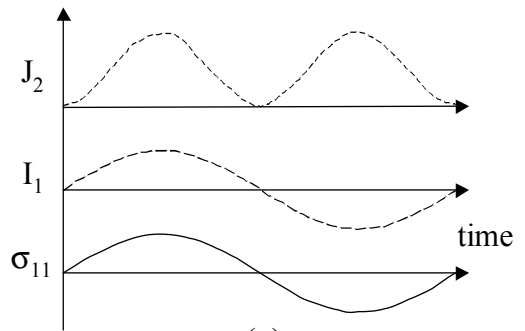

(a) the others, a cycle may occur on one channel but there is rarely a corresponding cycle at the same time period on another channel (figure 3).

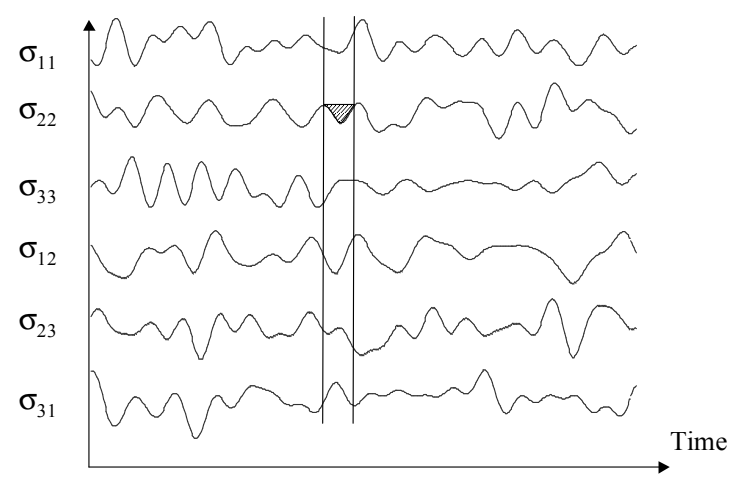

Figure 3. Problem of cycle's identification for a multiaxial variable amplitude stress states histoty.

The counting and identification of cycles need consequently the requirement of a counting variable which must be a correct representation of the stress states and of their evolution as a function of time $t$.

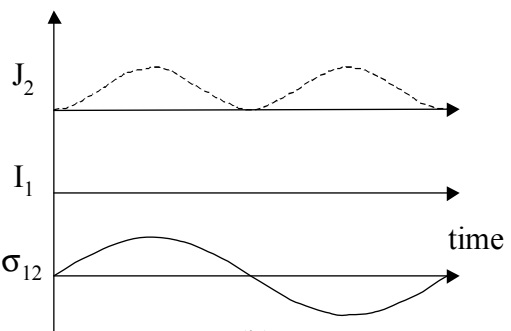

(b)

Figure 4. Evolution of invariants $J_{2}$ and $I_{1}$ : (a) during a pure torsion-compression cycle, (b) during a pure torsion cycle.

Stress or deviatory stress invariants are not representative of the evolution of stress states as a function of time enough to be considered as the counting variable. Figure 4 gives, for a pure tension-compression stress states cycle (respectively a pure torsion stress states cycle), the corresponding evolution of the second invariant $\mathrm{J}_{2}$ of the deviatory stress tensor and the first invariant $I_{1}$ of the stress tensor.

The first invariant $I_{1}$ is not suitable to count cycles for a pure torsion stress states as it remains equal to zero during the whole cycle (the hydrostatic pressure is equal to zero for such stress states). The second invariant $\mathrm{J}_{2}$ of the deviatory stress tensor is not usable too as in either tension or torsion cases; its frequency is twice the real frequency of the stress states. This variable would not allow one to identify the real cycles.

As the fatigue crack initiation on a given plane is induced by stresses that are acting on it, the counting variable has to be closely related to these stresses. The shear stress acting on a given plane may rotate in this plane during the stress history. Consequently two components are necessary to describe it properly and thus make the Rain flow counting be inapplicable from this point of view.

The normal stress $\sigma_{h h}$ acting on a considered physical plane that is defined by its unit normal vector $\vec{h}$ (figure 5) is proposed as the counting variable. Two angles $\varphi$ and $\gamma$ define the orientation of the unit vector $\vec{h}$ that is expressed by:

$$
\overrightarrow{\mathrm{h}}=\left(\begin{array}{rrrr}
\sin & \gamma & \cos & \varphi \\
\sin & \gamma & \sin & \varphi \\
& \cos & \gamma &
\end{array}\right)
$$

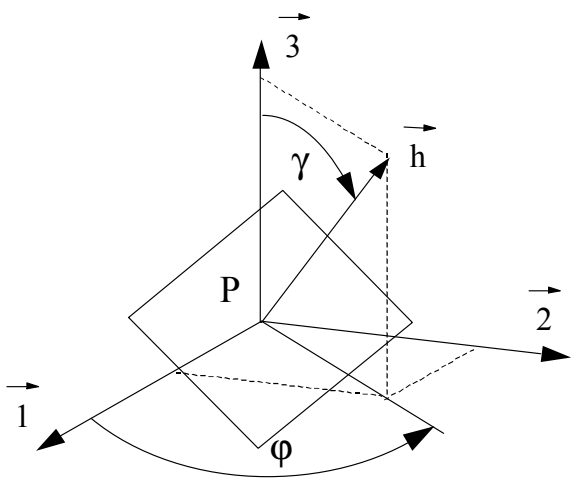

Figure 5. Definition of the unit normal vector $\overrightarrow{\boldsymbol{h}}$ to a physical plane. 
The identification of a cycle is governed by the fact that the counting variable does not remain constant when the stress tensor is changing it is to say when any of its six components is varying with respect to time. Otherwise no stress evolution is detected and the counting procedure can not identify any stress cycle. From this point of view, the identification of cycles is very similar to the uniaxial stress states cycle occurrence conditions.

When a cycle occurs within the counting variable history, the corresponding multiaxial stress cycle is extracted from the multiaxial stress states sequences (figure 6). The fatigue life of such a cycle is then determined by the use of a multiaxial stress criterion.



Figure 6. Identification of a multiaxial stress states cycle.

\section{Fatigue Life Prediction Method}

\subsection{Multiaxial Critical Plane Criterion}

A multiaxial fatigue criterion based on the critical plane concept is employed to assess the life of any multiaxial stress cycles [6]. For that purpose it has been extended from infinite fatigue lives (endurance limit) to finite ones. The formulation of the critical plane endurance criterion is given by equation (1). $\left[\sigma_{i j}(t)\right]$ represents the stress states cycle. $\sigma_{-1}, \sigma_{0}$ and $\tau_{-1}$ are the so-called fatigue limits of the material for a reversed tensile test (stress ratio $\mathrm{R}$ equal to -1 ), a reversed torsion test $(\mathrm{R}=-1)$ and a zero-to-maximum tensile test $(R=0)$ respectively. $E$ is the fatigue function of the criterion.

$$
\mathrm{E}\left(\left[\sigma_{\mathrm{ij}}(\mathrm{t})\right], \sigma_{-1}, \sigma_{0}, \tau_{-1}\right)=1
$$

The formulation of the criterion adapted to finite lives is:

$$
\mathrm{E}\left(\left[\sigma_{\mathrm{ij}}(\mathrm{t})\right], \sigma_{-1}(\mathrm{~N}), \sigma_{0}(\mathrm{~N}), \tau_{-1}(\mathrm{~N})\right)=1
$$

where $\sigma_{-1}(\mathrm{~N}), \sigma_{0}(\mathrm{~N})$ and $\tau_{-1}(\mathrm{~N})$ are the fatigue strengths of the material corresponding to $\mathrm{N}$ cycles for the same cyclic stress states as $\sigma_{-1}, \sigma_{0}$ and $\tau_{-1}$. Equation (3) indicates that the fatigue life of the material for the multiaxial cycle $\left[\sigma_{i j}(t)\right]$ is $\mathrm{N}$ cycles. For the endurance criterion, the fatigue life corresponds to the threshold of the endurance domain.

The fatigue function $\mathrm{E}$ of the criterion is obtained from searching the critical material plane. A time dependent fatigue indicator $E_{h}(t)$ is defined as a linear combination of the different mean and alternate stress components of the stress vector $\vec{\phi}_{\mathrm{h}}$ that is acting on the material plane which unit normal vector is denoted $\vec{h}$.

$$
E_{h}(t)=\frac{\left\|\vec{\tau}_{\text {ha }}(t)\right\|+\alpha(N) \sigma_{\text {hha }}(t)+\beta(N) \sigma_{\text {hhm }}}{\theta(N)}
$$

where $\vec{\tau}_{\text {ha }}(t)$ and $\sigma_{\text {hha }}(t)$ are the alternate shear stress vector and the alternate normal stress that are acting on the considered plane at time t. $\sigma_{\mathrm{hhm}}$ is the normal stress acting on that plane during the cycle.

$\vec{\tau}_{\text {ha }}(t)$ is obtained by building the surrounding circle to the so-called loading path which is constituted by the tip of the shear stress vector $\vec{\tau}_{\mathrm{h}}(\mathrm{t})$ during the cycle (figure 7). The loading path is generally a closed loop, especially for constant amplitude solicitations. The center $\mathrm{O}$ of the surrounding circle defines the mean shear stress vector $\vec{\tau}_{\mathrm{m}}(\mathrm{t})$ acting on the material plane during the cycle (it is generally assumed it has no contribution to the fatigue damage of the material thus it is not involved in the fatigue indicator $E_{h}(t)$ ). The alternate shear stress vector is stated as:

$$
\vec{\tau}_{\text {ha }}(t)=\vec{\tau}_{h}(t)-\vec{\tau}_{\text {hm }}
$$

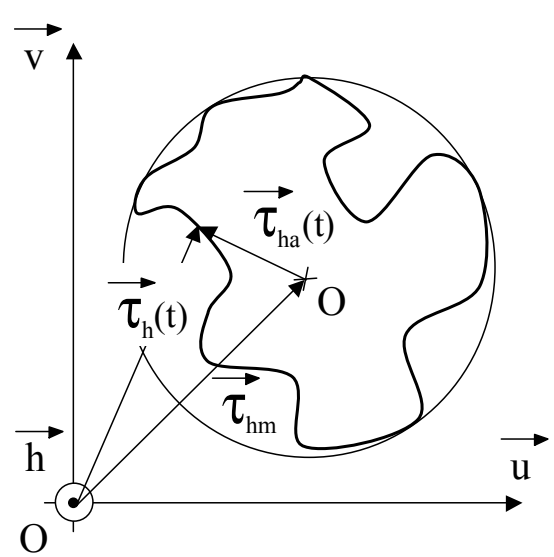

Figure 7. Definition of the mean and alternate components of the shear stress vector.

Finally the fatigue indicator $\mathrm{E}_{\mathrm{h}}$ of the criterion during a cycle for a given physical plane is obtained by:

$$
\mathrm{E}_{\mathrm{h}}=\operatorname{Max}_{\mathrm{t}} \mathrm{E}_{\mathrm{h}}(\mathrm{t})
$$

The critical plane criterion concept requires searching for the most damaged plane (denoted as the critical plane) during 
the considered stress cycle, it is to say the plane where $E_{h}$ reaches the highest value:

$$
\mathrm{E}_{\mathrm{h}}=\underset{\overrightarrow{\mathrm{h}}}{\operatorname{Max}} \mathrm{E}_{\mathrm{h}}
$$

The parameters $\alpha(\mathrm{N}), \beta(\mathrm{N})$ and $\theta(\mathrm{N})$ are used to establish the damage indicator of the criterion. They are obtained by stating that the multiaxial critical plane criterion is verified for the three basic fatigue tests up to $\mathrm{N}$ cycles that give the three fatigue strengths $\sigma_{-1}(\mathrm{~N}), \sigma_{0}(\mathrm{~N})$ and $\tau_{-1}(\mathrm{~N})$. For each one of these fatigue tests one has: $E=1$. Finally the parameters are expressed as:

$$
\alpha(N)=\frac{\frac{\tau_{-1}(\mathrm{~N})}{\sigma_{-1}(\mathrm{~N})}-\frac{1}{2}}{\sqrt{\frac{\tau_{-1}(\mathrm{~N})}{\sigma_{-1}(\mathrm{~N})}\left(1-\frac{\tau_{-1}(\mathrm{~N})}{\sigma_{-1}(\mathrm{~N})}\right)}} \theta(\mathrm{N})=\tau_{-1}(\mathrm{~N}) \sqrt{\alpha \mathrm{N})^{2}+1} \quad ; \beta(\mathrm{N})=\frac{2 \theta(\mathrm{N})}{\sigma_{0}(\mathrm{~N})}-\frac{\sigma_{0}(\mathrm{~N})}{8 \theta(\mathrm{N})}-\alpha(\mathrm{N})
$$

$\alpha(\mathrm{N}), \beta(\mathrm{N})$ and $\theta(\mathrm{N})$ describe in fact the sensitivity of the material to the alternate shear stress, to the alternate normal stress and to the mean normal stress. They determine the respective contributions of these stress components to the fatigue damage of the material. In the case where the three fatigue strengths vary with the same factor versus the number of cycles, these respective sensitivities of the material remain the same. Otherwise some of them may become predominant for the fatigue damage process depending on the microstructure of the material [7].

The fatigue life of the material for a given multiaxial cycle is determined by solving equation (2). An implicit algorithm is used to calculate the life N. It is based upon the meaning of the difference between the criterion fatigue function $\mathrm{E}$ and the theoretical value $(E=1)$ when the fatigue strength of the material submitted to the given multiaxial cycle is reached:

1. If $\mathrm{E}$ is greater than unity $(\mathrm{E}>1)$, the cycle has larger stress amplitude that what the material is able to endure $\mathrm{N}$ times. The real fatigue life is less than $\mathrm{N}$ cycles (reference life) that were used to make the first calculations of E. Parameters $\alpha, \beta$ and $\theta$ are recalculated for a smaller life $\mathrm{N}$ and a new fatigue

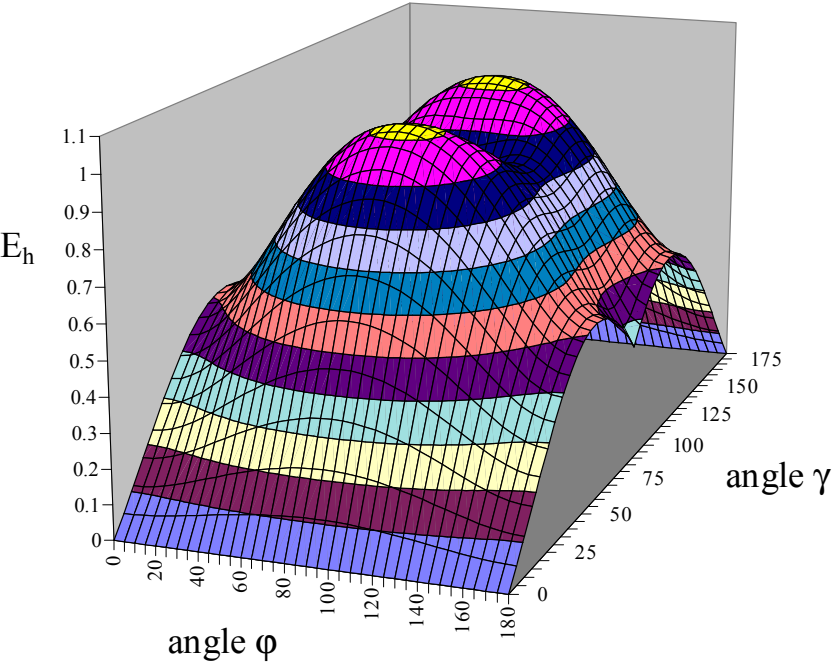

(a) function $\mathrm{E}$ is assessed.

2. In the other case $(E<1)$, the real fatigue life is greater than the reference one. Parameters $\alpha, \beta, \theta$ and the fatigue function $\mathrm{E}$ are calculated once again for a greater number of cycles.

It is to notice that this concept allows one to assess the real fatigue life of any material plane. As a matter of fact, all the possible material plane are not submitted to the same levels of shear and normal stresses. This induces various values of the damage indicator depending on the orientation of the considered plane as shown for the two following examples. Figure 8-a gives the distribution of the damage indicator $E_{h}$ for a fixed principal stress directions cycle. Figure 8-b gives the same representation for a cycle which principal stress directions rotate. The damage indicators were calculated in both cases for the number of cycles $\mathrm{N}_{\mathrm{D}}$ corresponding to the endurance threshold of the material. In other words $\alpha, \beta$ and $\theta$ were established by using the fatigue limits $\sigma_{-1}, \sigma_{0}$ and $\tau_{-1}$.

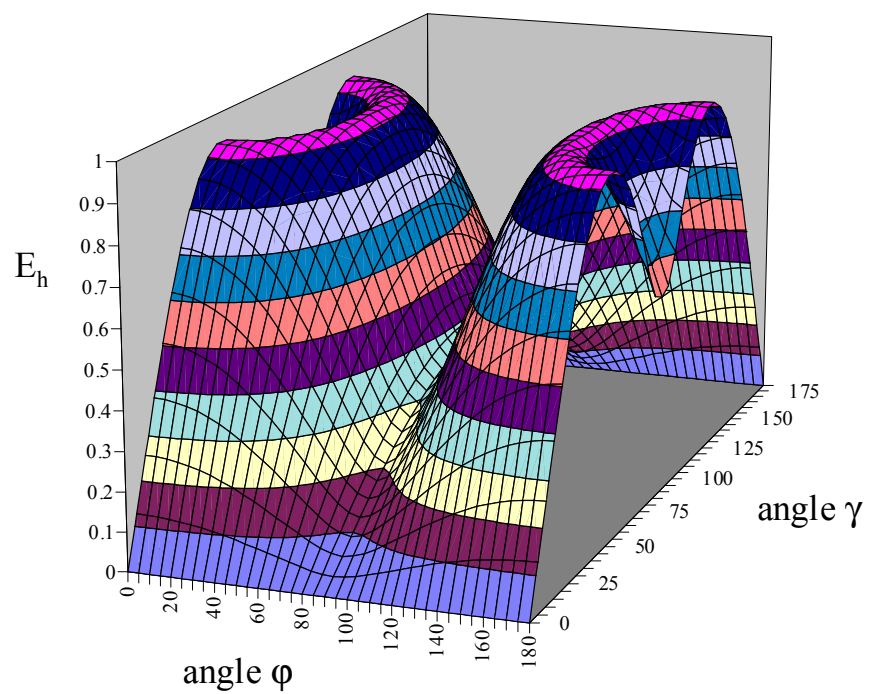

(b)

Figure 8. Distribution of the fatigue indicator $E_{h}:$ (a) for a constant principal stress directions cycle, b) for a rotating principal stress directions cycle. 
These two distributions were obtained with material data given in table 1:

Table 1. Material and stress states cycles data.

\begin{tabular}{|c|c|c|c|c|c|c|c|}
\hline & Material & Ref. & $\mathrm{N}_{\mathrm{D}}$ (cycles) & $\sigma_{-1}$ (Мра) & $\sigma_{0}(\mathbf{M P a})$ & $\tau_{-1}$ (MPa) & $\sigma_{i j}(t)$ \\
\hline Case (a) & $\mathrm{XC} 48$ & [8] & $10^{5}$ & 423 & 716 & 287 & $\begin{array}{l}\sigma_{11}=183+183 \sin (\omega t) \\
\sigma_{22}=367+367 \sin (\omega t)\end{array}$ \\
\hline Case (b) & $35 \mathrm{Cr} 4$ & [9] & $1.5 \cdot 10^{6}$ & 410 & 640 & 256 & $\sigma_{11}=380 \sin (\omega t) \sigma_{12}=95 \sin \left|\omega t+\frac{\pi}{2}\right|$ \\
\hline
\end{tabular}

$\varphi$ and $\gamma$ are the angles that define the orientation of the unit normal vector $\vec{h}$ to the considered plane.

The distribution of the damage indicator all over the possible material planes shows the more severe aspect of the multiaxial rotating principal stress directions cycle. When principal stress directions are fixed, a limited number of planes are critical. In the case of rotating principal stress directions, an infinite number of planes are equally critical. In this case of course the microstructure defects or weakness induce the site of the crack initiation among all these possible planes.

\subsection{Plane Per Plane Damage Cumulation}

The fatigue life of a material plane is derived by the method described above for all the cycles this material plane experiences. A damage law is used to assess the damage induced by each cycle and a cumulation rule allows one to obtain the amount of damage corresponding to the whole sequence.

Two damage rules may be used for this step of the method. The first one is the well known linear Miner's rule [8], the second one is the non linear law proposed by Lemaitre and Zhi Yong Huang [9]. This law gives the damage increase $\delta D$ due to $\delta \mathrm{N}$ identical uniaxial stress cycles defined by their amplitude $\sigma_{\mathrm{a}}$ and their mean value $\sigma_{\mathrm{m}}[17]$ as:

$$
\delta \mathrm{D}=\left[1-(1-\mathrm{D})^{\beta+1}\right]^{\alpha}\left[\frac{\sigma_{\mathrm{a}}}{\mathrm{M}_{0}\left(1-\mathrm{b} \sigma_{\mathrm{m}}\right)(1-\mathrm{D})}\right]^{\beta} \delta \mathrm{N}
$$

Where:

$$
\alpha=1-\mathrm{a} \frac{\sigma_{\mathrm{a}}-\sigma_{\mathrm{A}}\left(\sigma_{\mathrm{m}}\right)}{\mathrm{R}_{\mathrm{m}}-\sigma_{\mathrm{a}}-\sigma_{\mathrm{m}}} \text { if } \sigma_{\mathrm{a}}>\sigma_{\mathrm{A}}\left(\sigma_{\mathrm{m}}\right) \text { (large }
$$

$$
\alpha=1 \text { If } \sigma_{a}<\sigma_{A}\left(\sigma_{m}\right) \text { (small amplitude cycle), }
$$

$\sigma_{A}\left(\sigma_{m}\right)$ Is the material fatigue limit as a function of the mean stress. It is given by the endurance constant life diagram of the material and is expressed as:

$$
\sigma_{\mathrm{A}}=\sigma_{-1}\left(1-\mathrm{b} \sigma_{\mathrm{m}}\right)
$$

$\mathrm{a}, \mathrm{b}, \boldsymbol{\beta}$ and $\mathrm{M}_{0}$ are material parameters,

$\mathrm{R}_{\mathrm{m}}$ is the material ultimate tensile strength.

This non linear damage law allows small amplitude cycles to contribute to the material damage and takes into account the occurrence order of the cycles.

\section{First Validation of the Fatigue Life Prediction Method}

The first validation of the proposed method against experiments is realised with biaxial random stress states histories. The tests were carried out by Jan Papuga [10] in the laboratory of professor Macha in Opole (Poland), on cruciform specimen (figure 9) made of low carbon steel denoted 10HNAP. The tables 2 and 3 give respectively the chemical composition of the material and its mechanical

\begin{tabular}{|c|c|c|c|c|c|c|c|c|}
\hline Elements & $\mathbf{C}$ & Mn & $\mathbf{S i}$ & $\mathbf{P}$ & $\mathbf{S}$ & $\mathrm{Cr}$ & $\mathrm{Cu}$ & $\mathbf{N i}$ \\
\hline Contents $(\%)$ & 0.115 & 0.71 & 0.41 & 0.082 & 0.028 & 0.81 & 0.3 & 0.5 \\
\hline
\end{tabular}
static properties.

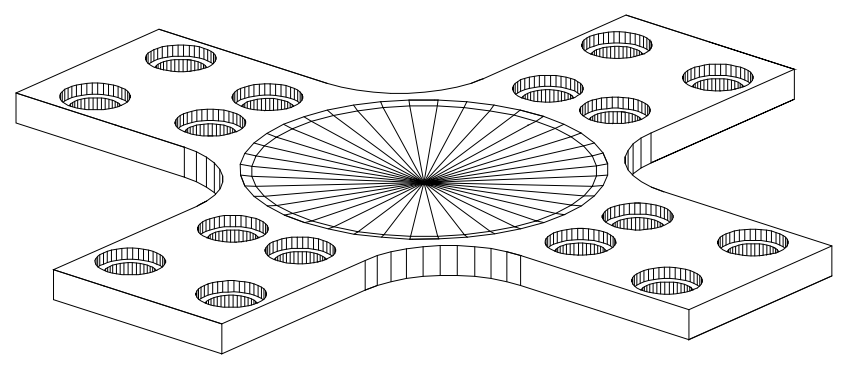

Figure 9. Cruciform specimen used for biaxial stress states fatigue tests.

Table 2. Chemical composition of the 10HNAP steel.

Table 3. Material mechanical static properties.

\begin{tabular}{llll}
\hline$\sigma_{\mathrm{e}}[\mathrm{MPa}]$ & $\mathrm{R}_{\mathrm{m}}[\mathrm{MPa}]$ & $\boldsymbol{v}$ & $\mathbf{E}[\mathbf{M P a}]$ \\
\hline 418 & 566 & 0.29 & 215000 \\
\hline
\end{tabular}

Three material crack initiation S-N curves $\sigma_{-1}(\mathrm{~N}), \sigma_{0}(\mathrm{~N})$ and $\tau_{-1}(\mathrm{~N})$ for respectively reversed tension-compression tests $(R=-1)$, zero to maximum tensile tests $(R=0)$ and reversed torsion tests $(\mathrm{R}=-1)$, are required. These material data are plotted on figures 10,11 and 12 . 


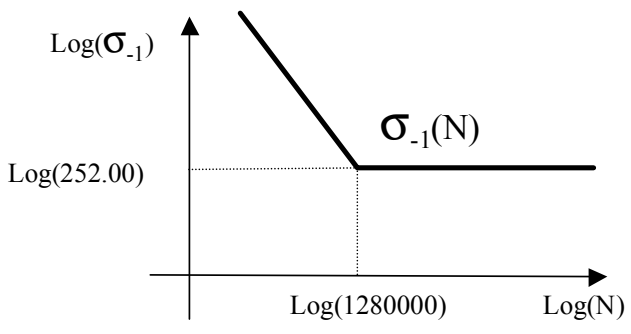

Figure 10. Fully reversed tension-compression $S-N$ curve $\sigma_{-1}(N)$.

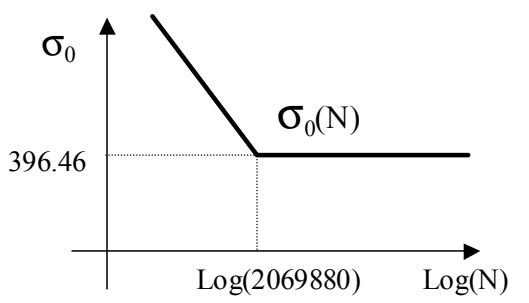

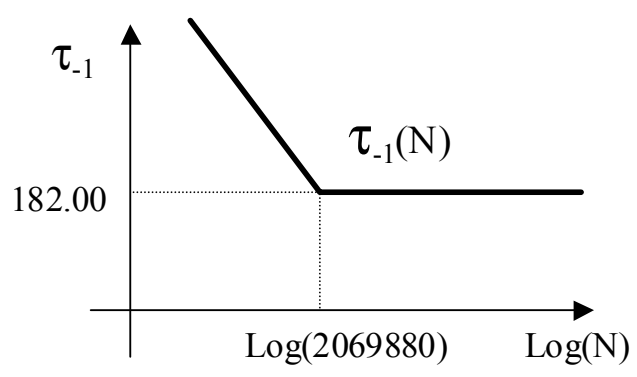

Figure 12. Fully reversed torsion $S-N$ curve $\tau_{-1}(N)$.

Fourteen sequences of biaxial stress states (figure 13) were tested. Experimental lives are compared against predicted ones, which were calculated by using both linear and non linear damage laws. Lives are expressed as the number of repetitions of sequences up to crack initiation. Predicted lives are plotted against experimental ones in figure 14 .

Figure 11. Zero to maximum tensile $S-N$ curve $\sigma_{0}(N)$.

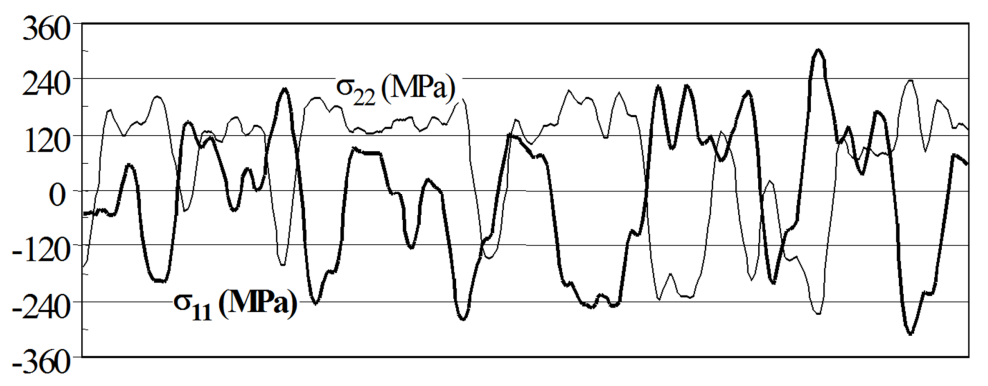

Figure 13. Biaxial stress states sequences (sample).

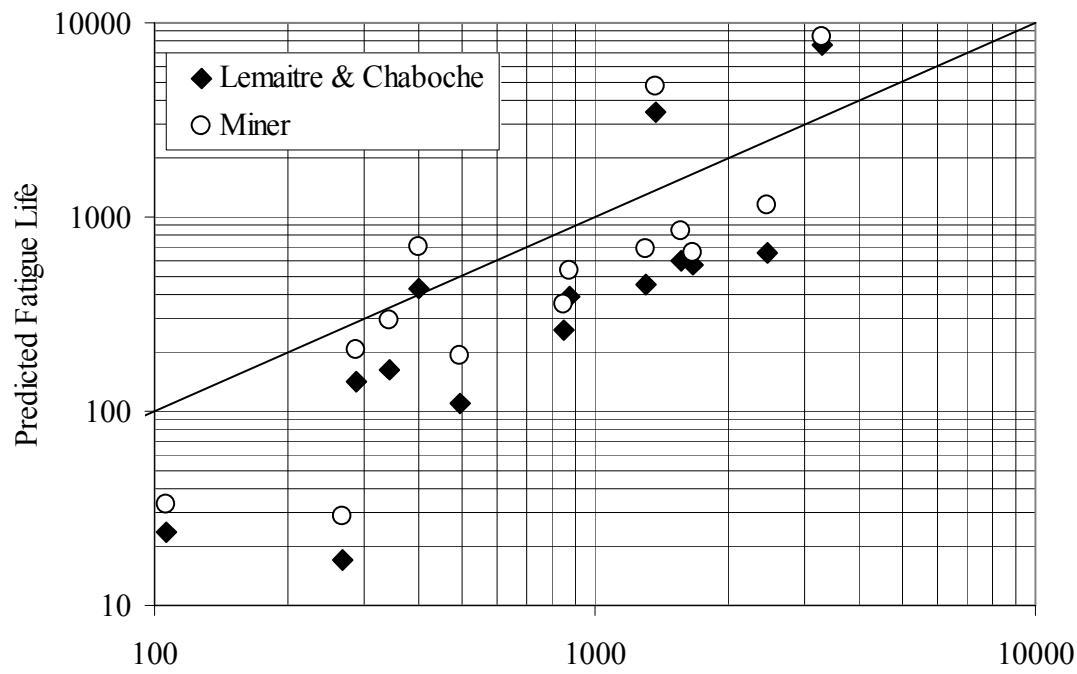

Experimental Fatigue Life

Figure 14. Experimental results against predicted assessments.

Predicted fatigue lives are generally conservative, especially when the non linear damage law is used. This comes from the fact that according to that non linear law small amplitude cycles bring their own contribution to the damage and make decrease the fatigue lives. The average ratio between experimental lives and predicted ones are equal to 2.2 and 3.4 for Miner's rule and Lemaitre and Chaboche non linear damage law.
The determination of the critical plane gives the fatigue life of the material up to crack initiation and also the orientation of the expected fracture plane corresponding to each sequence. The distribution of the fatigue damage of physical planes can be plotted versus the orientation of their normal vector defined by the two angles $\varphi$ and $\gamma$. The figure 15 gives an example of such a distribution obtained for one treated sequence. A double symmetry with respect to 
$\varphi=90^{\circ}$ and $\gamma=90^{\circ}$ is observed because of the pure biaxial stress states of the sequences. The distributions have identical shapes for Miner's rule (right side) and Lemaitre and Chaboche's rule (left side).

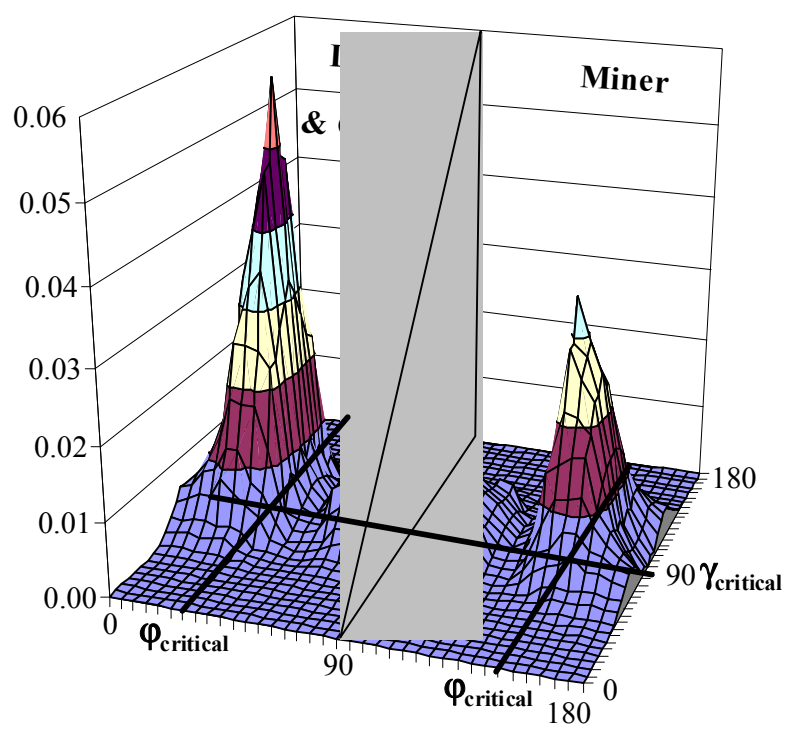

Figure 15. Distribution of the damage versus physical planes orientations according to the non linear / linear damage laws.

A life assessment requires the examination of all the possible material planes. Practically, a selected number of physical planes are examined and the calculation procedure is repeated for each of them. An optimization of the orientations of the investigated planes has been realized [11] so that their normal vectors are equally distributed in a three dimensional space and has allowed a strong reduction of time calculations. Suitable algorithms were also developed for the determination of the alternate shear stress vector $\vec{\tau}_{\text {ha }}(t)$ by building the smallest surrounding circle to the loading path. The exact geometrical solution is now rapidly found for any kind of loading path. The calculation times are now admissible with respect to current design request from an industrial point of view.

\section{Conclusion}

A new fatigue life prediction method suitable for any kind of multiaxial variable amplitude stress states history is proposed. It is a stress approach based upon a plane per plane damage distribution, directly related to the stresses experienced by these material planes. The identification of multiaxial cycles is processed for any plane by considering the normal stress to this plane as the counting parameter. A multiaxial fatigue criterion extended from endurance to finite lives allows one to assess the life of the cycle with accounting for the six components of the stress states tensor. Linear or non linear damage laws are usable to express and make the cumulation of damage versus time. The procedure allows the assessment both of the crack initiation plane and the fatigue life of the material. A first validation of the life prediction method is realized by the way of fourteen biaxial random stress states histories issued from tests carried out on cruciform steel specimens. The average values of the ratio between experimental lives and expected ones indicate conservative assessments of 2.2 by using Miner's damage rule and 3.4 with Lemaitre and Chaboche damage law.

\section{References}

[1] Carpinteri A., Spagnoli A., Critical plane criterion for fatigue life calculation: time and frequency domain formulations, $3 \mathrm{rd}$ International Conference on Material and Component Performance under Variable Amplitude Loading, VAL2015, 2015 pp. 518- 523.

[2] Wang C. H., Brown M. W., Life Prediction Tecliniques for Variable Amplitude Multiaxial Fatigue, Journal of Engineering Materials and Technology, 1996, Vol. 118/367.

[3] Zheng-Yong Yu, Shun-Peng Zhu, A New Energy-Critical Plane Damage Parameter for Multiaxial Fatigue Life Prediction of Turbine www.mdpi.com/journal/materials, 2017.

[4] Nicholas R. Gates, Ali Fatemi, Multiaxial variable amplitude fatigue life analysis using the critical plane approach, Part I: Un-notched specimen experiments and life estimations, International Journal of Fatigue, 2017 pp. 283-295.

[5] Yingyu Wang, Critical Plane Approach to Multiaxial Variable Amplitude Fatigue Loading, Frattura ed Integrità Strutturale, 2015 pp. 345-356.

[6] Wang, Y. and Susmel, L., The Modified Manson-Coffin Curve Method to estimate fatigue lifetime under complex constant and variable amplitude multiaxial fatigue loading. International Journal of Fatigue, 2016, pp. 135-149.

[7] Bannantine, J. A. and Socie, D. F., A variable amplitude multiaxial fatigue life prediction method. In Fatigue under Biaxial and Multiaxial Loading, ESIS 10, ed. K. Kussmaul, D. McDiarmid and D. F. Socie. Mechanical Engineering Publications, London, 1991, pp. 35-51.

[8] Miner, M. A., Cumulative damage in fatigue, Journal of Applied Mechanics, 1945, pp. 159-164.

[9] Zhi Yong Huang, Danièle Wagner, Claude Bathias, Jean Louis Chaboche, Cumulative fatigue damage in low cycle fatigue and gigacycle fatigue for low carbon-manganese steel, 2018.

[10] Jan Papuga, Quest for fatigue limit prediction under multiaxial loading, Procedia Engineering, 5th Fatigue Design Conference, Fatigue Design 2013.

[11] Robert, J. L., Fogue, M. and Bahuaud, J., Fatigue life prediction under periodical or random multiaxial stress states. In Automation in Fatigue and Fracture: Testing and Analysis, ASTM STP 1231, ed. C. Amzallag. ASTM, Philadelphia, 1994, pp. 369-387. 